\title{
A Strong Convergence Theorem for Total Asymptotically Pseudocontractive Mappings in Hilbert Spaces
}

\author{
Chuan Ding ${ }^{1}$ and Jing Quan ${ }^{2}$ \\ ${ }^{1}$ School of Economic Mathematics, Southwestern University of Finance and Economics, \\ Chengdu 610074, China \\ ${ }^{2}$ Department of Mathematics, Yibin University, Yibin, Sichuan 644007, China
}

Correspondence should be addressed to Jing Quan, quanjingcq@163.com

Received 19 May 2012; Accepted 27 August 2012

Academic Editor: Irena Lasiecka

Copyright (C) 2012 C. Ding and J. Quan. This is an open access article distributed under the Creative Commons Attribution License, which permits unrestricted use, distribution, and reproduction in any medium, provided the original work is properly cited.

Demiclosedness principle for total asymptotically pseudocontractive mappings in Hilbert spaces is established. The strong convergence to a fixed point of total asymptotically pseudocontraction in Hilbert spaces is obtained based on demiclosedness principle, metric projective operator, and hybrid iterative method. The main results presented in this paper extend and improve the corresponding results of Zhou (2009), Qin, Cho, and Kang (2011) and of many other authors.

\section{Introduction}

Throughout this article we assume that $H$ is a real Hilbert space, whose inner product and norm are denoted by $\langle\cdot, \cdot\rangle$ and $\|\cdot\|$, respectively; $C$ is a nonempty closed convex subset of $H ; \mathbb{N}$ and $\mathbb{R}^{+}$denote the natural number set and the set of nonnegative real numbers, respectively. Let $T: C \rightarrow C$ be a nonlinear mapping; $F(T)$ denotes the set of fixed points of mapping $T$. We use " $\rightarrow$ " to stand for strong convergence and " $\rightarrow$ " for weak convergence.

Recently, the iterative approximation of fixed points for asymptotically pseudocontractive mappings, total asymptotically pseudocontractive mappings in Hilbert, or Banach spaces has been studied extensively by many authors, see for example [1-5].

The asymptotically pseudocontractions and total asymptotically pseudo-contractions are defined as follows.

Definition 1.1 (see [3]). T:C $\rightarrow C$ is said to be asymptotically pseudocontraction if there exists a sequence $\left\{k_{n}\right\}$ of positive real numbers with $k_{n} \geq 1, \lim _{n \rightarrow+\infty} k_{n}=1$, such that

$$
\left\langle T^{n} x-T^{n} y, x-y\right\rangle \leq k_{n}\|x-y\|^{2}
$$


holds; in [4], $T: C \rightarrow C$ is said to be total asymptotically pseudo-contraction if there exists sequences $\left\{\mu_{n}\right\},\left\{v_{n}\right\}$ with $\mu_{n}, v_{n} \rightarrow 0$ as $n \rightarrow \infty$ and strictly increasing continuous functions $\psi: \mathbb{R}^{+} \rightarrow \mathbb{R}^{+}$with $\psi(0)=0$, such that

$$
\left\langle T^{n} x-T^{n} y, x-y\right\rangle \leq\|x-y\|^{2}+\mu_{n} \psi(\|x-y\|)+v_{n}, \quad n \in \mathbb{N}
$$

holds; Zhou [3, page 3144] have proved the following Theorem.

\section{Theorem Zhou}

Let $C$ be a bounded and closed convex subset of a real Hilbert space $H$. Let $T: C \rightarrow C$ be a uniformly $L$-Lipschitzian asymptotically pseudo-contraction with a fixed point. Suppose the control sequence $\alpha_{n}$ is chosen so that $\alpha_{n} \in[a, b]$, for some $a, b \in(0,1 /(1+L))$. Let $x_{n}$ be a sequence generated in the following manner:

$$
\begin{gathered}
x_{0} \in C, \quad \text { chosen arbitrarily; } n \geq 0, \\
y_{n}=\left(1-\alpha_{n}\right) x_{n}+\alpha_{n} T^{n} x_{n}, \\
C_{n}=\left\{z \in C: \alpha_{n}\left[1-(1+L) \alpha_{n}\right]\left\|x_{n}-T^{n} x_{n}\right\|^{2}\right. \\
\left.\leq\left\langle x_{n}-z, y_{n}-T^{n} y_{n}\right\rangle+\left(k_{n}-1\right)(\operatorname{diam} C)^{2}\right\}, \\
Q_{n}=\left\{z \in C:\left\langle x_{n}-z, x_{0}-x_{n}\right\rangle\right\} \geq 0, \\
x_{n+1}=P_{C_{n} \cap Q_{n}} x_{0} .
\end{gathered}
$$

Then the iterative sequence $\left\{x_{n}\right\}$ converges strongly to $P_{F(T)} x_{0}$.

Qin et al. [5] introduced the class of total asymptotically pseudocontractive mappings in Hilbert spaces and established the following weak convergence theorem of fixed points.

\section{Theorem Qin}

Let $C$ be a bounded and closed convex subset of a real Hilbert space $H$. Let $T: C \rightarrow C$ be a uniformly $L$-Lipschitzian total asymptotically pseudo-contractive mapping. Assume that $F(T)$ is nonempty and there exist positive constants $M$ and $M^{*}$ such that $\psi(\lambda) \leq M^{*} \lambda^{2}$ for all $\lambda \geq M$. Let $x_{n}$ be a sequence generated in the following manner:

$$
\begin{gathered}
x_{1} \in C, \\
y_{n}=\left(1-\beta_{n}\right) x_{n}+\beta_{n} T^{n} x_{n}, \\
x_{n+1}=\left(1-\alpha_{n}\right) x_{n}+\alpha_{n} T^{n} y_{n}, \quad \forall n \geq 1,
\end{gathered}
$$

where $\alpha_{n}$ and $\beta_{n}$ are sequences in $(0,1)$. Assume that the following restrictions are satisfied:

(a) $\sum_{n=1}^{\infty} \mu_{n}<\infty$ and $\sum_{n=1}^{\infty} v_{n}<\infty$.

(b) $a<\alpha_{n}<\beta_{n}<b$ for some $a>0$ and some $b \in\left(0, L^{-2}\left[\sqrt{1+L^{2}}-1\right]\right)$.

Then the iterative sequence $\left\{x_{n}\right\}$ converges weakly to fixed point of $T$. 
The purpose of this article is to prove the strong convergence for total asymptotically pseudo-contraction in Hilbert spaces. The results presented in the article improve and extend the corresponding results of Zhou [3], Qin et al. [5], and many other authors.

\section{Preliminaries}

A mapping $T: C \rightarrow C$ is said to be uniformly $L$-Lipschitzian if there exists some $L>0$ such that

$$
\left\|T^{n} x-T^{n} y\right\| \leq L\|x-y\|
$$

holds for all $x, y \in C$ and for all $n \in \mathbb{N}$. Let $C$ be a nonempty closed convex subset of a real Hilbert space $H$. For every point $x \in H$ there exists a unique nearest point in $C$, denoted by $P_{C} x$, such that $\left\|x-P_{C} x\right\| \leq\|x-y\|$ holds for all $y \in C$, where $P_{C}$ is called the metric projection of $H$ onto $C$.

In order to prove the results of this article, we will need the following lemmas.

Lemma 2.1 (see [6]). Let $C$ be a nonempty closed convex subset of a real Hilbert space $H$. Given $x \in H$ and $z \in C$, then $z=P_{C} x$ if and only if there holds the relation

$$
\langle x-z, y-z\rangle \leq 0, \quad \forall y \in C \text {. }
$$

Lemma 2.2 (see [6]). Let $C$ be a nonempty closed convex subset of a real Hilbert space $H$ and $P_{C}$ : $H \rightarrow C$ the metric projection from $H$ onto $C$. Then the following inequality holds:

$$
\left\|y-P_{C} x\right\|^{2}+\left\|x-P_{C} x\right\|^{2} \leq\|x-y\|^{2}, \quad \forall x \in H, y \in C
$$

\section{Main Results}

Theorem 3.1 (demiclosedness principle). Let $C$ be a nonempty bounded and closed convex subset of a real Hilbert space $H$. Let $T: C \rightarrow C$ be a uniformly L-Lipschitzian and total asymptotically pseudo-contraction. Suppose there exists $M^{*}>0$, such that $\psi\left(\xi_{n}\right) \leq M^{*} \xi_{n}$, then $I-T$ is demiclosed at zero, where I is the identical mapping.

Proof. Assume that $\left\{x_{n}\right\} \subset C$, with $x_{n} \rightarrow x$ and $x_{n}-T x_{n} \rightarrow 0$ as $n \rightarrow \infty$. We want to prove $x \in C$ and $x=T x$. Since $C$ is a closed convex subset of $H$, so $x \in C$. In the following we prove $x=T x$.

Now we choose $\alpha \in(0,1 /(1+L))$ and let $y_{\alpha, m}=(1-\alpha) x+\alpha T^{m} x$ for arbitrary fixed $m \geq 1$. Because $T$ is uniformly $L$-Lipschitzian, we have

$$
\begin{aligned}
\left\|x_{n}-T^{m} x_{n}\right\| & \leq\left\|x_{n}-T x_{n}\right\|+\left\|T x_{n}-T^{2}(t) x_{n}\right\|+\cdots+\left\|T^{m-1} x_{n}-T^{m} x_{n}\right\| \\
& \leq m L\left\|x_{n}-T x_{n}\right\| \\
& \longrightarrow 0, \quad \text { as } n \longrightarrow \infty .
\end{aligned}
$$


Since $T$ is total asymptotically pseudo-contraction, we have

$$
\begin{aligned}
\left\langle x-y_{\alpha, m},\left(I-T^{m}\right) y_{\alpha, m}\right\rangle & \\
= & \left\langle x-x_{n},\left(I-T^{m}\right) y_{\alpha, m}\right\rangle+\left\langle x_{n}-y_{\alpha, m},\left(I-T^{m}\right) y_{\alpha, m}\right\rangle \\
= & \left\langle x-x_{n},\left(I-T^{m}\right) y_{\alpha, m}\right\rangle+\left\langle x_{n}-y_{\alpha, m},\left(I-T^{m}\right) x_{n}\right\rangle \\
& +\left\langle x_{n}-y_{\alpha, m},\left(I-T^{m}\right) y_{\alpha, m}-\left(I-T^{m}\right) x_{n}\right\rangle \\
\leq & \left\langle x-x_{n},\left(I-T^{m}\right) y_{\alpha, m}\right\rangle+\left\langle x_{n}-y_{\alpha, m},\left(I-T^{m}\right) x_{n}\right\rangle \\
& +\mu_{m} \psi\left(\left\|x_{n}-y_{\alpha, m}\right\|\right)+v_{m} .
\end{aligned}
$$

By assumption $x_{n} \rightarrow x, x_{n}-T x_{n} \rightarrow 0$ and $\left\|x_{n}-T^{m} x_{n}\right\| \rightarrow 0$ as $n \rightarrow \infty$, we have

$$
\begin{aligned}
\langle x- & \left.y_{\alpha, m},\left(I-T^{m}\right) y_{\alpha, m}\right\rangle \\
& \leq \mu_{m} \psi\left(\left\|x_{n}-y_{\alpha, m}\right\|\right)+v_{m} \\
& \leq \mu_{m} M^{*}\left\|x_{n}-y_{\alpha, m}\right\|+v_{m} \\
& \leq \mu_{m} M^{*}(\operatorname{diam} C)+v_{m} .
\end{aligned}
$$

By the $L$-Lipschitz of $T$ and the definition of $y_{\alpha, m}$, we have

$$
\begin{gathered}
\left\langle x-y_{\alpha, m},\left(I-T^{m}\right) x-\left(I-T^{m}\right) y_{\alpha, m}\right\rangle \\
\leq(1+L)\left\|x-y_{\alpha, m}\right\|^{2} \\
\leq(1+L) \alpha^{2}\left\|x-T^{m} x\right\|^{2} .
\end{gathered}
$$

Thus we have

$$
\begin{aligned}
\left\|x-T^{m} x\right\|^{2} & =\left\langle x-T^{m} x, x-T^{m} x\right\rangle=\frac{1}{\alpha}\left\langle x-y_{\alpha, m}, x-T^{m} x\right\rangle \\
& =\frac{1}{\alpha}\left\langle x-y_{\alpha, m}, x-T^{m} x-\left(y_{\alpha, m}-T^{m} y_{\alpha, m}\right)\right\rangle+\frac{1}{\alpha}\left\langle x-y_{\alpha, m},\left(y_{\alpha, m}-T^{m} y_{\alpha, m}\right)\right\rangle \\
& \leq \alpha(1+L)\left\|x-T^{m} x\right\|^{2}+\frac{1}{\alpha}\left\langle x-y_{\alpha, m}, y_{\alpha, m}-T^{m} y_{\alpha, m}\right\rangle \\
& \leq \alpha(1+L)\left\|x-T^{m} x\right\|^{2}+\frac{1}{\alpha}\left(\mu_{m} M^{*}(\operatorname{diam} C)+v_{m}\right),
\end{aligned}
$$

which implies that

$$
\alpha[1-\alpha(1+L)]\left\|x-T^{m} x\right\|^{2} \leq \mu_{m} M^{*}(\operatorname{diam} C)+v_{m}, \quad \forall m \in \mathbb{N} .
$$

When $m \rightarrow \infty, \mu_{m}, v_{m} \rightarrow 0$, so we have $\left\|x-T^{m} x\right\| \rightarrow 0, m \rightarrow \infty$, that is, $T^{m} x \rightarrow x, m \rightarrow \infty$, so $T^{m+1} x \rightarrow T x, m \rightarrow \infty$. By the continuity of $T$, we have $T x=x$. 
Theorem 3.2. Let $C$ be a nonempty bounded and closed convex subset of a real Hilbert space H. Let $T: C \rightarrow C$ be a uniformly L-Lipschitzian and total asymptotically pseudo-contraction. Suppose there exists $M^{*}>0$, such that $\psi\left(\xi_{n}\right) \leq M^{*} \xi_{n}$, then $F(T)$ is a closed convex subset of $C$.

Proof. Since $T$ is uniformly $L$-Lipschitzian continuous, $F(T)$ is closed. We need to show that $F(T)$ is convex. We let $p_{1}, p_{2} \in F(T)$, and $p=t p_{1}+(1-t) p_{2}$ for $t \in(0,1)$. We take $\alpha \in$ $(0,1 /(1+L))$ and let $y_{\alpha, n}=(1-\alpha) p+\alpha T^{n} p, n \in \mathbb{N}$. Then for any $z \in F(T)$, we have

$$
\begin{aligned}
\left\|p-T^{n} p\right\|^{2} & =\left\langle p-T^{n} p, p-T^{n} p\right\rangle=\frac{1}{\alpha}\left\langle p-y_{\alpha, n}, p-T^{n} p\right\rangle \\
& =\frac{1}{\alpha}\left\langle p-y_{\alpha, n}, p-T^{n} p-\left(y_{\alpha, n}-T^{n} y_{\alpha, n}\right)\right\rangle+\frac{1}{\alpha}\left\langle p-y_{\alpha, n}, y_{\alpha, n}-T^{n} y_{\alpha, n}\right\rangle \\
& \leq \frac{1+L}{\alpha}\left\|p-y_{\alpha, n}\right\|^{2}+\frac{1}{\alpha}\left\langle p-z, y_{\alpha, n}-T^{n} y_{\alpha, n}\right\rangle+\frac{1}{\alpha}\left\langle z-y_{\alpha, n}, y_{\alpha, n}-T^{n} y_{\alpha, n}\right\rangle \\
& \leq \frac{1+L}{\alpha}\left\|p-y_{\alpha, n}\right\|^{2}+\frac{1}{\alpha}\left\langle p-z, y_{\alpha, n}-T^{n} y_{\alpha, n}\right\rangle+\frac{1}{\alpha}\left(\mu_{n} M^{*}(\operatorname{diam} C)+v_{n}\right) \\
& \leq \alpha(1+L)\left\|p-T^{n} p\right\|^{2}+\frac{1}{\alpha}\left\langle p-z, y_{\alpha, n}-T^{n} y_{\alpha, n}\right\rangle+\frac{1}{\alpha}\left(\mu_{m} M^{*}(\operatorname{diam} C)+v_{m}\right)
\end{aligned}
$$

This implies that

$$
\alpha[1-\alpha(1+L)]\left\|p-T^{n} p\right\|^{2} \leq\left\langle p-z, y_{\alpha, n}-T^{n} y_{\alpha, n}\right\rangle+\mu_{m} M^{*}(\operatorname{diam} C)+v_{m} .
$$

Now we take $z=p_{1}, p_{2}$, multiplying $t$ and $1-t$ on both sides of above inequality, respectively, and adding up, and we can get

$$
\alpha[1-\alpha(1+L)]\left\|p-T^{n} p\right\|^{2} \leq \mu_{m} M^{*}(\operatorname{diam} C)+v_{m} .
$$

By $n \rightarrow \infty$, we get $T^{n} p \rightarrow p$. Since $T$ is continuous, we have $T^{n+1} p \rightarrow T p$ as $n \rightarrow \infty$, so that $p=T p$.

Theorem 3.3. Let $C$ be a bounded and closed convex subset of a real Hilbert space $H$. Let $T: C \rightarrow C$ be a uniformly L-Lipschitzian and total asymptotically pseudo-contraction. Suppose there exists $M^{*}>$ 0 , such that $\psi\left(\xi_{n}\right) \leq M^{*} \xi_{n}, F(T) \neq \emptyset, \alpha_{n}$ is a sequence in $[a, b]$, where $a, b \in(0,1 /(1+L))$. Let $x_{n}$ be a sequence generated by

$$
\begin{gathered}
x_{1}=x \in C, \quad \forall n \in \mathbb{N}, \\
y_{n}=\left(1-\alpha_{n}\right) x_{n}+\alpha_{n} T^{n} x_{n}, \\
H_{n}=\left\{z \in C: \alpha_{n}\left[1-(1+L) \alpha_{n}\right]\left\|x_{n}-T^{n} x_{n}\right\|^{2} \leq\left\langle x_{n}-z, y_{n}-T^{n} y_{n}\right\rangle+\zeta_{n}\right\}, \\
W_{n}=\left\{z \in C:\left\langle x_{n}-z, x-x_{n}\right\rangle\right\} \geq 0, \\
x_{n+1}=P_{H_{n} \cap W_{n}} x,
\end{gathered}
$$

where $\zeta_{n}=\mu_{m} M^{*}(\operatorname{diam} C)+v_{m}$, then the iterative sequence $\left\{x_{n}\right\}$ converges strongly to $P_{F(T)} x$ in $C$. 
Proof. We divide the proof into seven steps.

(I) $P_{F(T)}$ is well defined for every $x \in C$.

By Theorem 3.2, we know $F(T)$ is closed and convex subset of $C$. Moreover, by our assumption that $F(T)$ is nonempty, therefore, $P_{F(T)} x$ is well defined for every $x \in C$.

(II) $H_{n}$ and $W_{n}$ are closed and convex for all $n \in \mathbb{N}$.

From the definitions of $W_{n}$ and $H_{n}$, it is obvious that $H_{n}$ and $W_{n}$ are closed and convex for each $n \in \mathbb{N}$. We omit the details.

(III) We prove $F(T) \subset H_{n} \cap W_{n}$ for each $n \in \mathbb{N}$.

We first show $F(T) \subset H_{n}$. Let $z \in F(T)$, by (3.10), and the uniform $L$-Lipschitz continuity of $T$ and the total asymptotical pseudo-contractiveness of $T$, we have

$$
\begin{aligned}
\left\|x_{n}-T^{n} x_{n}\right\|^{2}= & \left\langle x_{n}-T^{n} x_{n}, x_{n}-T^{n} x_{n}\right\rangle \\
= & \frac{1}{\alpha_{n}}\left\langle x_{n}-y_{n}, x_{n}-T^{n} x_{n}\right\rangle \\
= & \frac{1}{\alpha_{n}}\left\langle x_{n}-y_{n}, x_{n}-T^{n} x_{n}-\left(y_{n}-T^{n} y_{n}\right)\right\rangle+\frac{1}{\alpha_{n}}\left\langle x_{n}-y_{n},\left(y_{n}-T^{n} y_{n}\right)\right\rangle \\
\leq & \frac{1+L}{\alpha_{n}}\left\|x_{n}-y_{n}\right\|^{2}+\frac{1}{\alpha_{n}}\left\langle x_{n}-z, y_{n}-T^{n} y_{n}\right\rangle+\frac{1}{\alpha_{n}}\left(\mu_{n} M^{*}(\operatorname{diam} C)+v_{n}\right) \\
= & (1+L) \alpha_{n}\left\|x_{n}-T^{n} x_{n}\right\|^{2}+\frac{1}{\alpha_{n}}\left\langle x_{n}-z, y_{n}-T^{n} y_{n}\right\rangle \\
& +\frac{1}{\alpha_{n}}\left(\mu_{n} M^{*}(\operatorname{diam} C)+v_{n}\right) .
\end{aligned}
$$

This implies that

$$
\alpha_{n}\left[1-\alpha_{n}(1+L)\right]\left\|x_{n}-T^{n} x_{n}\right\|^{2} \leq\left\langle x_{n}-z, y_{n}-T^{n} y_{n}\right\rangle+\left(\mu_{n} M^{*}(\operatorname{diam} C)+v_{n}\right) .
$$

This shows that $z \in H_{n}$ for all $n \in \mathbb{N}$. So $F(T) \subset H_{n}$ for all $n \in \mathbb{N}$. Next we prove $F(T) \subset W_{n}$ for all $n \in \mathbb{N}$. By induction, for $n=1$, we have $F(T) \subset C=W_{1}$. Assume that $F(T) \subset W_{n}$. Since $x_{n+1}$ is the projection of $x$ onto $H_{n} \cap W_{n}$, by Lemma 2.1, we have

$$
\left\langle x_{n+1}-z, x-x_{n+1}\right\rangle \geq 0,
$$

for any $z \in H_{n} \bigcap W_{n}$, by the definition of $W_{n+1}$, this shows that $z \in W_{n+1}$. So $F(T) \subset H_{n} \bigcap W_{n}$, for all $n \in \mathbb{N}$.

(IV) We prove that $\lim _{n \rightarrow \infty}\left\|x_{n}-x\right\|$ exists.

From (3.10) and Lemma 2.1, we have $x_{n}=P_{w_{n}} x$, this with $x_{n+1} \in W_{n+1}$ show $\left\|x_{n}-x\right\| \leq$ $\left\|x_{n+1}-x\right\|$, for all $n \in \mathbb{N}$. As $z \in F(T) \subset W_{n}$, we also have $\left\|x_{n}-x\right\| \leq\|z-x\|$, for all $n \in \mathbb{N}$. Consequently, $\lim _{n \rightarrow \infty}\left\|x_{n}-x\right\|$ exists and $\left\{x_{n}\right\}$ is bounded.

(V) We prove that $\left\|x_{n+1}-x_{n}\right\| \rightarrow 0$ as $n \rightarrow \infty$. 
By Lemma 2.2, we have

$$
\left\|x_{n+1}-x_{n}\right\|^{2} \leq\left\|x_{n+1}-x\right\|^{2}-\left\|x_{n}-x\right\|^{2} \longrightarrow 0,
$$

as $n \rightarrow \infty$.

(VI) Now we prove $\left\|x_{n}-T x_{n}\right\| \rightarrow 0$ as $n \rightarrow \infty$.

It follows from $\left\|x_{n+1}-x_{n}\right\| \rightarrow 0$ as $n \rightarrow \infty, x_{n+1} \subset H_{n},\left\{y_{n}\right\}$ is bounded, $\left\{T^{n} y_{n}\right\}$ is bounded, and $\alpha_{n} \in(a, b)$ that

$$
\begin{aligned}
\alpha_{n}[1 & \left.-\alpha_{n}(1+L)\right]\left\|x_{n}-T^{n} x_{n}\right\|^{2} \\
& \leq\left\langle x_{n}-z, y_{n}-T^{n} y_{n}\right\rangle+\left(\mu_{n} M^{*}(\operatorname{diam} C)+v_{n}\right) \\
& \leq\left\|x_{n}-z\right\|\left\|y_{n}-T^{n} y_{n}\right\|+\left(\mu_{n} M^{*}(\operatorname{diam} C)+v_{n}\right) \longrightarrow 0, \quad n \longrightarrow \infty .
\end{aligned}
$$

So $\left\|x_{n}-T^{n} x_{n}\right\| \rightarrow 0$ as $n \rightarrow \infty$. Additional

$$
\begin{aligned}
\left\|x_{n}-T x_{n}\right\| \leq & \left\|x_{n+1}-x_{n}\right\|+\left\|T^{n+1} x_{n+1}-x_{n+1}\right\| \\
& +\left\|T^{n+1} x_{n}-T^{n+1} x_{n+1}\right\|+\left\|T x_{n}-T^{n+1} x_{n}\right\| \\
\leq & (L+1)\left\|x_{n+1}-x_{n}\right\|+\left\|T^{n+1} x_{n+1}-x_{n+1}\right\|+L\left\|x_{n}-T^{n} x_{n}\right\| .
\end{aligned}
$$

So $\left\|x_{n}-T x_{n}\right\| \rightarrow 0$ as $n \rightarrow \infty$.

(VII) Finally, we prove $x_{n} \rightarrow P_{F(T)} x$ as $n \rightarrow \infty$.

Let $x_{n_{k}}$ be a subsequence of $x_{n_{k}}$ such that $x_{n_{k}} \rightarrow \hat{x} \in C$, then by Theorem 3.1, we have $\hat{x} \in F(T)$. We let $\omega \in P_{F(T)} x$. For any $n \in \mathbb{N}, x_{n+1}=P_{H_{n} \cap W_{n}} x$ and $\omega \in P_{F(T)} x \subset H_{n} \cap W_{n}$, so we get $\left\|x_{n+1}-x\right\| \leq\|\omega-x\|$.

On the other hand, from the weak lower semicontinuity of the norm, we have

$$
\begin{aligned}
\|\widehat{x}-x\|^{2} & =\|\widehat{x}\|^{2}-2\langle\widehat{x}, x\rangle+\|x\|^{2} \\
& \leq \liminf _{n \rightarrow \infty}\left(\left\|x_{n_{k}}\right\|^{2}-2\left\langle\left\|x_{n_{k}}\right\|^{2}, x\right\rangle+\|x\|^{2}\right) \\
& =\liminf _{n \rightarrow \infty}\left\|x_{n_{k}}-x\right\|^{2} \\
& \leq \limsup _{n \rightarrow \infty}\left\|x_{n_{k}}-x\right\|^{2} \\
& \leq\|\omega-x\|^{2} .
\end{aligned}
$$

From the definition of $P_{F(T)} x$, we obtain $\widehat{x}=\omega$ and hence lim $\sup _{n \rightarrow \infty}\left\|x_{n_{k}}-x\right\|^{2}=\|\omega-x\|^{2}$. So we have lim $\sup _{k \rightarrow \infty}\left\|x_{n_{k}}\right\|=\|\omega\|$. Thus we obtain that $x_{n_{k}}$ converges strongly to $P_{F(T)} x$. Since $x_{n_{k}}$ is an arbitrary weakly convergent sequence of $x_{n}$, we can conclude that $x_{n}$ converges strongly to $P_{F(T)} x$. This completes the proof of Theorem 3.3. 
Remark 3.4. Theorem 3.3 extends the main results of Zhou [3] and improves the main results of Qin et al. [5] and of many other authors.

\section{Acknowledgments}

The authors are grateful to the referees for their helpful and useful comments. This research is partially supported by the Fundamental Research Funds for the Central Universities (JBK120117), by the National Research Foundation of Yibin University (No. 2011B07) and by Scientific Research Fund Project of SiChuan Provincial Education Department (No. 12ZB345).

\section{References}

[1] S. S. Chang, "Some results for asymptotically pseudo-contractive mappings and asymptotically nonexpansive mappings," Proceedings of the American Mathematical Society, vol. 129, no. 3, pp. 845-853, 2001.

[2] M. O. Osilike and B. G. Akuchu, "Common fixed points of a finite family of asymptotically pseudocontractive maps," Fixed Point Theory and Applications, vol. 2, pp. 81-88, 2004.

[3] H. Zhou, "Demiclosedness principle with applications for asymptotically pseudo-contractions in Hilbert spaces," Nonlinear Analysis, vol. 70, no. 9, pp. 3140-3145, 2009.

[4] X. Qin, J. K. Kim, and T. Wang, "On the convergence of implicit iterative processes for asymptotically pseudocontractive mappings in the intermediate sense," Abstract and Applied Analysis, vol. 2011, Article ID 468716, 18 pages, 2011.

[5] X. Qin, S. Y. Cho, and S. M. Kang, "A weak convergence theorem for total asymptotically pseudocontractive mappings in Hilbert spaces," Fixed Point Theory and Applications, vol. 2011, Article ID 859795, 11 pages, 2011.

[6] G. Marino and H.-K. Xu, "Weak and strong convergence theorems for strict pseudo-contractions in Hilbert spaces," Journal of Mathematical Analysis and Applications, vol. 329, no. 1, pp. 336-346, 2007. 


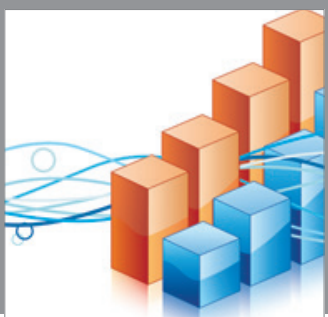

Advances in

Operations Research

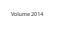

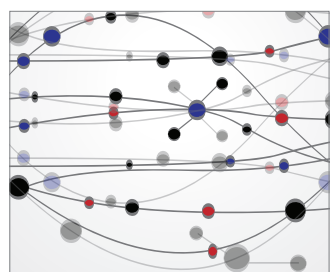

\section{The Scientific} World Journal
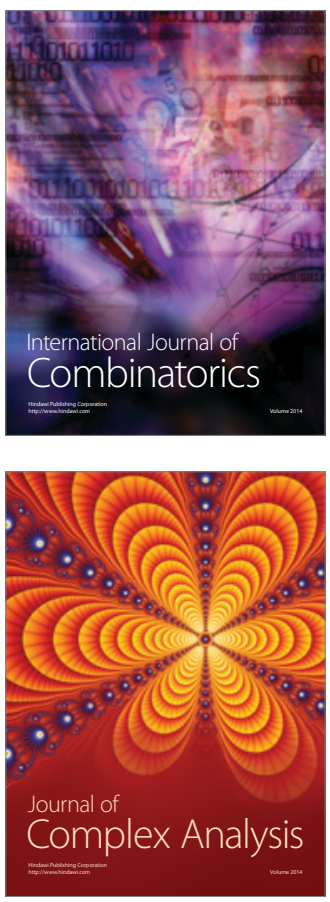

International Journal of

Mathematics and

Mathematical

Sciences
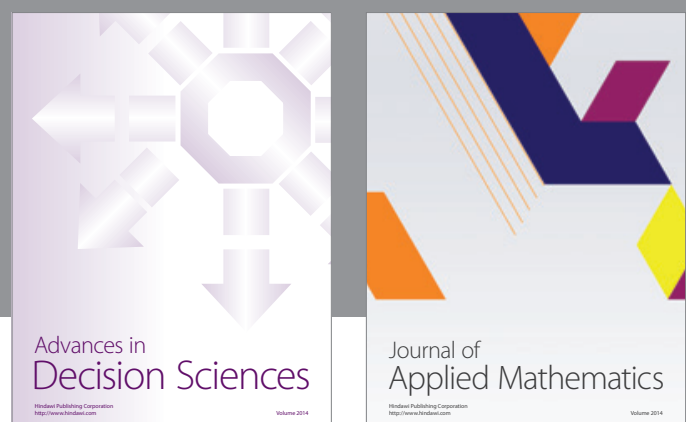

Journal of

Applied Mathematics
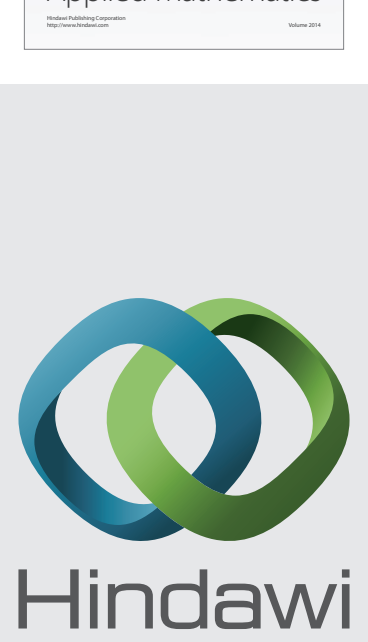

Submit your manuscripts at http://www.hindawi.com
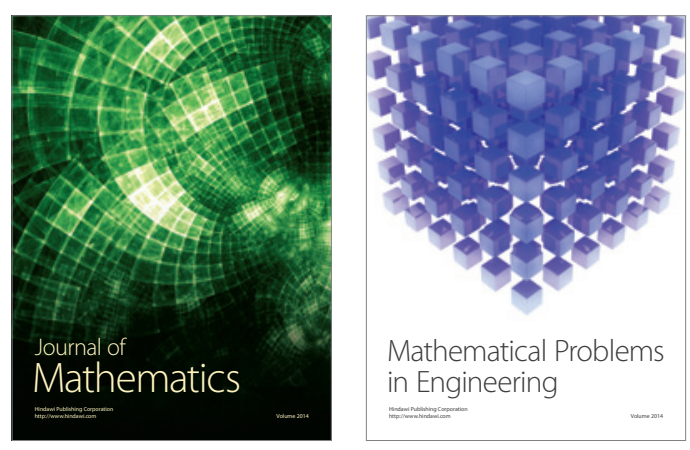

Mathematical Problems in Engineering
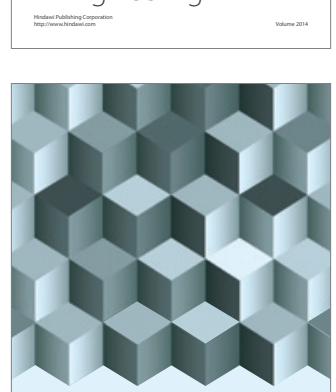

Journal of

Function Spaces
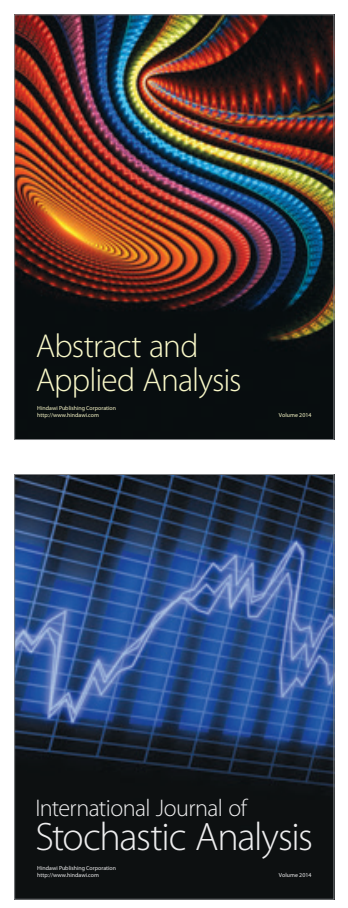

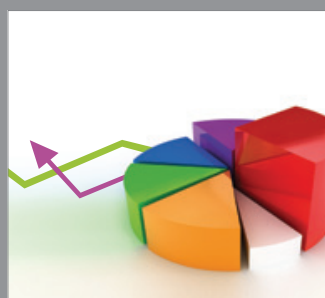

ournal of

Probability and Statistics

Promensencen
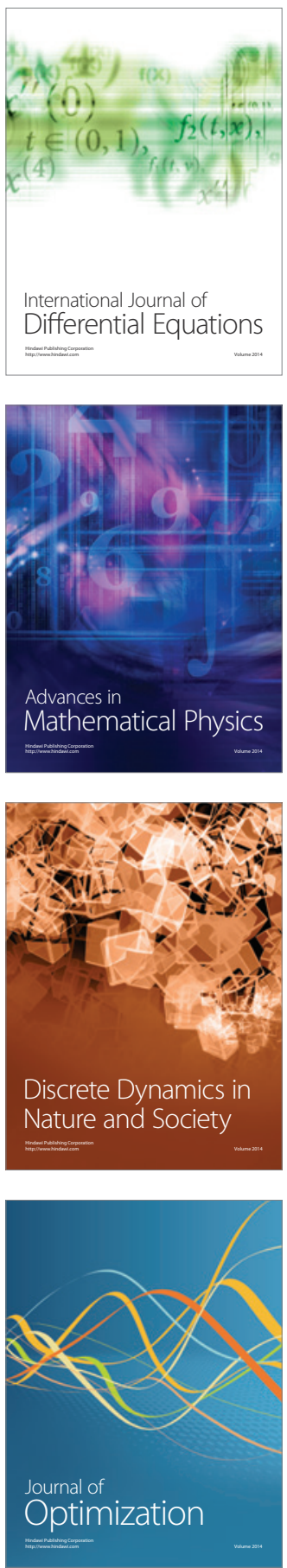